\title{
Effects of non-surgical periodontal therapy on serum inflammatory factor high-sensitive C-reactive protein, periodontal parameters and renal biomarkers in patients with chronic periodontitis and chronic kidney disease
}

\author{
Kirti Suresh Vachhani ${ }^{1,2, A-D}$, Neeta Vijay Bhavsar ${ }^{2, E, F}$ \\ ${ }^{1}$ Department of Dentistry, Faculty of Medicine, Gujarat University, Ahmedabad, India \\ ${ }^{2}$ Department of Periodontology, Government Dental College and Hospital, Ahmedabad, India \\ A - research concept and design; $B$ - collection and/or assembly of data; $C$ - data analysis and interpretation; \\ $D$ - writing the article; $E$ - critical revision of the article; $F$ - final approval of the article
}

Address for correspondence

Kirti Suresh Vachhani, PhD Scholar

E-mail: dr.kirtidulani@gmail.com

Funding sources

None declared

Conflict of interest

None declared

Acknowledgements

The authors would like to thank Dr. Sujal Parkar, MDS, PhD, assistant professor at the Department of Public Health Dentistry of Siddhpur Dental College and Hospital, India, for consulting on the statistical analysis.

Received on January 21, 202

Reviewed on March 27, 2021

Accepted on April 22, 2021

Published online on November 23, 2021

Cite as

Vachhani KS, Bhavsar NV. Effects of non-surgical periodontal therapy on serum inflammatory factor high-sensitive C-reactive protein, periodontal parameters and renal biomarkers in patients with chronic periodontitis and chronic kidney disease. Dent Med Probl. 2021;58(4):489-498. doi:10.17219/dmp/136034

DOI

$10.17219 / \mathrm{dmp} / 136034$

Copyright

○) 2021 by Wroclaw Medical University

This is an article distributed under the terms of the

Creative Commons Attribution 3.0 Unported License (CC BY 3.0)

(https://creativecommons.org/licenses/by/3.0/).

\section{Abstract}

Background. Chronic kidney disease (CKD) is associated with significant morbidity and mortality, and there are various risk factors for this disease. Although the association between CKD and periodontal disease (PD) has been reported in various cross-sectional studies, longitudinal intervention studies are scarce.

Objectives. This study aimed to evaluate the effects of non-surgical periodontal therapy (NSPT) on periodontal clinical parameters, serum inflammatory factor high-sensitivity C-reactive protein (hs-CRP) and renal biomarkers in patients with CKD and chronic periodontitis (CP).

Material and methods. A total of 80 patients with confirmed CKD aged 22-65 years, attending the Institute of Kidney Diseases Research (entre (IKDRC) in Ahmedabad, India, and referred to the Government Dental College and Hospital, Ahmedabad (GDCHA), were enrolled in this study. The patients were divided into 2 groups: group 1 received NSPT, including scaling and root planing (SRP), as well as oral hygiene instructions; and group 2 received oral hygiene instructions without NSPT. Periodontal clinical parameters, such as probing pocket depth (PPD), clinical attachment loss (CAL), bleeding on probing $(B O P)$, the periodontal inflamed surface area (PISA) score, and the Simplified Oral Hygiene Index (OHI-S), were recorded. Biomarkers, including hs-CRP, the estimated glomerular filtration rate (eGFR) and the urine albumin-to-creatinine ratio (UACR), were obtained from medical records. The comparisons of periodontal parameters, hs-CRP and renal biomarkers within and between the groups were performed at baseline, and 3 and 6 months after treatment.

Results. The periodontal parameter scores as well as the serum levels of hs-CRP and UACR significantly decreased while eGFR significantly increased in group 1 after treatment as compared to baseline $(p<0.001)$. Six months after treatment, group 1 showed significantly lower values than group 2 for periodontal parameters, the serum levels of hs-CRP and renal biomarkers except for eGFR, which improved and increased $(p<0.001)$.

Conclusions. Periodontitis is an important source of chronic inflammation and the treatment of periodontitis can hinder systemic inflammation in CKD patients. Non-surgical periodontal therapy resulted in improved periodontal health, with significant decreases in hs-CRP and UACR, and an increase in eGFR in CKD patients with CP in comparison with CKD patients not receiving NSPT.

Keywords: chronic renal insufficiency, glomerular filtration rate, periodontal disease, urine albumin-tocreatinine ratio 


\section{Introduction}

There is a well-known saying that "oral health is equal to overall health". The oral cavity is the intersection of dentistry and medicine, semi-independent fields that share the common goal of improving the health and quality of life of patients. Since the 1980s, research has considered the possibility that poor oral health substantially affects overall health. ${ }^{1}$ Chronic periodontitis (CP) is the most common oral inflammatory condition studied in this context. According to the 2010 Global Burden of Disease (GBD) Study, CP is the $6^{\text {th }}$ most prevalent condition, affecting $10.8 \%(95 \%$ uncertainty interval (UI): 10.1-11.6), i.e., 743 million people aged 15-99 worldwide. ${ }^{2}$ Chronic periodontitis is caused by dysbiotic oral biofilm that destroys the supporting connective tissues; a cascade of inflammatory immune responses fails to resolve the dysfunction and the dysregulated chronic inflammation ensues in a susceptible host. This results in the formation of periodontal pockets with chronically ulcerated pocket epithelium. ${ }^{3}$ In severe disease cases, the surface area of the ulcerated epithelium can be as large as $40 \mathrm{~cm}^{2}$, which increases microbial tissue invasion and perpetuates systemic inflammation accompanied by a rise in inflammatory biomarkers in serum, such as C-reactive protein (CRP) and interleukin (IL)-6. ${ }^{4}$

The course of non-communicable diseases, like atherosclerosis, myocardial infarction, non-hemorrhagic cerebrovascular disease, and diabetes mellitus, or adverse pregnancy outcomes can be related to the progression and treatment of periodontal disease (PD). ${ }^{5}$ This concept has been expanded to include systemic diseases, such as rheumatoid arthritis, malignant neoplasia and chronic kidney disease (CKD). ${ }^{5}$ Chronic kidney disease remains a prevalent public health problem. In 2017, the global prevalence of CKD was estimated at 9.1\% (697.5 million cases), with over a third of CKD patients living in 2 countries, namely China (132.3 million) and India (115.1 million). ${ }^{6}$ Chronic kidney disease is defined as either the occurrence of kidney damage or a decreased kidney function, wherein the estimated glomerular filtration rate (eGFR) amounts to $<60 \mathrm{~mL} / \mathrm{min} / 1.73 \mathrm{~m}^{2}$ for 3 months or longer. ${ }^{7}$ Chronic kidney disease is largely preventable and treatable. The trend of an increasing incidence of CKD as a direct cause of global morbidity and mortality, and also as an important risk factor for cardiovascular diseases (CVD) poses major challenges for both healthcare and the economy in the near future.

The association between CP and CKD has been studied widely since the 2000s and CP is now considered a novel, non-traditional risk factor for CKD. ${ }^{8,9}$ Chronic periodontitis may have its share in CVD-related mortality in CKD patients. ${ }^{9}$ However, prospective and interventional studies on this association with long follow-up periods are scarce. Thus, the present study was conducted to address this research gap by evaluating the effects of non-surgical periodontal therapy (NSPT) on clinical parameters, serum inflammatory factor high-sensitivity C-reactive protein (hs-CRP) and renal biomarkers in patients with CKD and CP.

\section{Material and methods}

A randomized, controlled, parallel-group, single-center pilot study was conducted from July 2018 to June 2019 .

\section{Ethical approval and consent}

Before the commencement of this study, the research protocol was submitted to the institutional ethics committee at Government Dental College and Hospital, Ahmedabad (GDCHA), India (IEC GDCH/S.6/2018, dated March 15, 2018), and subsequently approved. This study was registered in the Clinical Trials Registry - India (CTRI trial No. REF/2019/09/028096, dated September 14, 2019). The study was conducted in accordance with the 2020 Declaration of Helsinki and written informed consent was obtained from all patients.

\section{Participants and eligibility criteria}

Patients suffering from CKD, attending the outpatient department of the Institute of Kidney Diseases and Research Centre (IKDRC) in Ahmedabad, India, and referred to the Department of Periodontology of GDCHA were enrolled in this study. The inclusion criteria were as follows: male or female patients aged 18 years or older; patients with high-risk CKD, already receiving treatment for CKD at IKDRC, with eGFR $<60 \mathrm{~mL} / \mathrm{min} / 1.73 \mathrm{~m}^{2}$ for 3 months or longer, and the urine albumin-to-creatinine ratio (UACR) $\geq 70 \mathrm{mg} / \mathrm{gCr}$ on 3 consecutive occasions; patients having generalized moderate-to-severe periodontitis, i.e., with a minimum cumulative probing pocket depth (PPD) of $40 \mathrm{~mm}$ (defined as the sum of the greatest PPDs measured for each tooth, excluding PPDs $<5 \mathrm{~mm}$ ); and patients who provided informed consent to participate. The exclusion criteria were as follows: patients suffering from end-stage renal disease (ESRD) and requiring treatment with renal replacement therapy (RRT); patients receiving immunosuppressive medications; patients with a history of infective endocarditis or a heart valve replaced/repaired with prosthetic material; patients who underwent any periodontal treatment in the previous year; and patients who could not be subjected to periodontal treatment, e.g., with severe bleeding disorders that contraindicate periodontal treatment. 


\section{Randomization and allocation}

After considering the eligibility criteria, a total of 100 patients were enrolled. Randomization was based on a simple randomization method, wherein the patients enrolled on days 1-15 of the month were assigned to group 1 (test group) and those enrolled on days 16-30/31 of the month (16-28 for February 2019) were assigned to group 2 (control group) (Fig. 1).

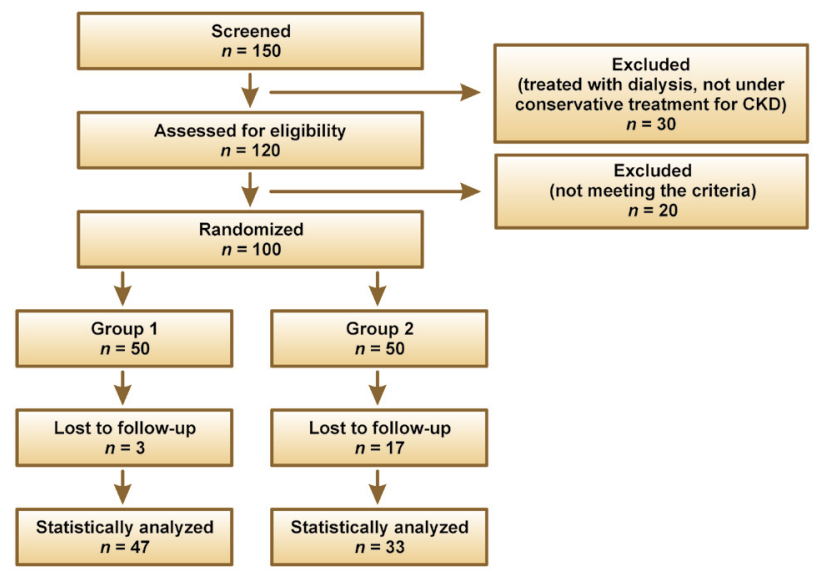

Fig. 1. Flowchart of the study

Group 1 patients received intensive NSPT, including supra/subgingival scaling and root planing (SRP) under local anesthesia, as well as oral hygiene instructions. Teeth with a poor prognosis and PPD $>8 \mathrm{~mm}$, grade III mobility in lateral and vertical directions, and/or retained infected root pieces were extracted during the baseline visit. The patients were supported with a maintenance program for a period of 6 months. Oral hygiene instructions were reinforced at each appointment. The patients were provided with a manual toothbrush and fluoridated toothpaste for maintaining good oral hygiene.

Group 2 patients received only oral hygiene instructions without any NSPT. The patients were monitored for the following 6 months at 3-month intervals.

All interventions were provided by the principal investigator, who was blinded to the group allocation. The patients were followed up to assess the effects of the interventions at 3 and 6 months after the intervention.

\section{Clinical examination and data collection}

The data was collected with regard to 3 sections. Section 1: Demographic details, including age, gender, the socio-economic status (SES) assessed by the Kuppuswamy scale ${ }^{10}$ doing exercise, the smoking status, and the body mass index (BMI), were collected through personal interviews with the patients. Section 2: Details of medical conditions, including systolic and diastolic blood pressure, renal biomarkers, like eGFR and UACR, and other biochemical values, like glycated hemoglobin (HbA1c), total cholesterol, triglycerides, and hs-CRP, were retrieved from the patients' medical records. Section 3: The clinical examination was carried out by a trained examiner to assess periodontal parameters, including the oral hygiene status assessed with the Simplified Oral Hygiene Index (OHI-S), ${ }^{11} \mathrm{PPD}$, the position of the gingival margin in terms of relative clinical attachment loss (CAL), and bleeding on probing (BoP) measured at 6 different sites (mesiobuccal, distobuccal, buccal, palatal/lingual, mesiolingual, and distolingual) for each tooth using a pressuresensitive probe (Bluedent India, Chennai, India) with a standardized pressure of $20 \mathrm{~g}$. The periodontal inflamed surface area (PISA), which reflects the amount of inflamed periodontal tissue in square millimeters, was calculated for each patient by entering the PPD, CAL and BoP measurements into a spreadsheet accessed via www. parsprototo.info. ${ }^{4}$

High-sensitivity assay techniques, such as immunonephelometry, immunoturbidimetry, high-sensitivity enzyme-linked immunosorbent assay (ELISA), and resonant acoustic profiling (RAP), can detect CRP with a sensitivity range of $0.01-10 \mathrm{mg} / \mathrm{L}$. In the present study, immunoturbidimetry was used, in which the immune complexes formed in the solution scatter light in proportion to their size, shape and concentration. Turbidimeters measure the reduction of incidence light due to reflection, absorption or scatter. What is measured in this procedure is the rate of decrease in the intensity (increase in the absorbance) of the light transmitted through the particles suspended in the solution, which is due to the complexes being formed during the immunological reaction between CRP in the patient's serum and the rabbit anti-CRP antibodies coating the latex particles. For this method, the AU400/400e/480, AU600/640/640e/680 and AU2700/5400 analyzers (Beckman Coulter, Brea, USA) were used with reagents, including glycine buffer $(100 \mathrm{mmol} / \mathrm{L})$ and latex particles coated with rabbit anti-CRP antibodies $(<0.5 \%)$.

All data related to medical conditions and periodontal parameters was assessed at 3 different time points, i.e., at baseline (before any interventions), at 3 months and at 6 months.

\section{Statistical analysis}

After data collection, the data was encoded and entered into Microsoft ${ }^{\circledR}$ Excel $^{\circledR} 2019$ (Microsoft Corporation, Redmond, USA). The data was checked for normality with the Shapiro-Wilk test. As the data deviated significantly from a normal distribution $(p<0.05)$, non-parametric tests were applied for the analysis. Demographic variables were reported as mean $(M)$ and standard deviation $(S D)$ or as number $(n)$ and percentage (\%), and compared using the $X^{2}$ test. Student's $t$ test was used to compare the mean values for quantitative data. The data for clinical variables 
was presented as $M$ and $S D$ or as median $(M e)$ and interquartile range $(I Q R)$. The values for different time points were compared within both groups using Friedman's test. In case of significant results of Friedman's test, Scheffe's test was used for post hoc pairwise comparisons. The Mann-Whitney $U$ test was applied for comparisons between the 2 groups. For all tests, the level of statistical significance was set at $p<0.05$. The statistical analysis was performed using the IBM SPSS Statistics for Windows software, v. 22.0 (IBM Corp., Armonk, USA).

\section{Results}

Out of the total 100 patients selected for this study, 80 completed 6 months of follow-up. During the 6-month follow-up period, 14 patients developed ESRD and required preparation for dialysis (the creation of an arteriovenous (AV) fistula), 4 died during the course of treatment, and 2 did not attend follow-up. Therefore, 47 patients from group 1 and 33 patients from group 2 completed the full study (Fig. 1, Table 1).

Demographic characteristics for groups 1 and 2 are shown in Table 1 . The mean patient age was $50.62 \pm 10.35$ years and $50.45 \pm 10.87$ years in group 1 and group 2 , respectively. There were no statistically significant differences in demographic variables between the groups $(p>0.05)$.

There were highly significant differences within groups 1 and 2 when the median values of periodontal parameters, systemic inflammatory marker hs-CRP and renal biomarkers were compared across the study time points $(p<0.001)$. The intragroup comparisons with regard to different time points also exhibited significant differences in the post hoc test $(p<0.05)$ (Table 2$)$.

Table 1. Demographic characteristics and some clinical data of the study participants

\begin{tabular}{|c|c|c|c|c|c|}
\hline \multicolumn{3}{|c|}{ Variables } & $\begin{array}{l}\text { Group } 1 \\
(n=47)\end{array}$ & $\begin{array}{l}\text { Group } 2 \\
(n=33)\end{array}$ & $p$-value \\
\hline \multicolumn{3}{|l|}{ Age [years] } & $50.62 \pm 10.35$ & $50.45 \pm 10.87$ & 0.950 \\
\hline \multirow{2}{*}{ Gender $^{b}$} & \multicolumn{2}{|c|}{ male } & 35 (43.8) & $24(30.0)$ & \multirow{2}{*}{1.000} \\
\hline & \multicolumn{2}{|c|}{ female } & $12(15.0)$ & $9(11.3)$ & \\
\hline \multirow{4}{*}{ SESC } & \multicolumn{2}{|c|}{ upper lower } & $4(5.0)$ & $1(1.3)$ & \multirow{4}{*}{0.070} \\
\hline & \multicolumn{2}{|c|}{ lower middle } & $11(13.8)$ & $8(10.0)$ & \\
\hline & \multicolumn{2}{|c|}{ upper middle } & $25(31.3)$ & $24(30.0)$ & \\
\hline & \multicolumn{2}{|c|}{ upper } & $7(8.80)$ & $0(0.0)$ & \\
\hline \multirow{2}{*}{ Doing exercise ${ }^{b}$} & \multicolumn{2}{|c|}{ yes } & $8(10.0)$ & $12(15.0)$ & \multirow{2}{*}{0.080} \\
\hline & & & 39 (48.8) & $21(26.3)$ & \\
\hline \multirow{3}{*}{ Smoking ${ }^{c}$} & \multicolumn{2}{|c|}{ never } & $23(28.8)$ & $19(23.8)$ & \\
\hline & \multicolumn{2}{|c|}{ former } & $19(23.8)$ & $11(13.8)$ & 0.750 \\
\hline & \multicolumn{2}{|c|}{ current } & $5(6.3)$ & $3(3.8)$ & \\
\hline \multirow{6}{*}{ Comorbidity $^{b}$} & \multirow{2}{*}{ diabetes } & yes & $21(26.3)$ & $11(13.8)$ & \multirow{2}{*}{0.430} \\
\hline & & no & $26(32.5)$ & $22(27.5)$ & \\
\hline & \multirow{2}{*}{ CVS disorder } & yes & $11(13.8)$ & $9(11.3)$ & \multirow{2}{*}{0.890} \\
\hline & & no & $36(45.0)$ & $24(30.0)$ & \\
\hline & \multirow{2}{*}{ CNS disorder } & yes & $2(2.5)$ & $0(0.0)$ & \multirow{2}{*}{0.640} \\
\hline & & no & $45(56.3)$ & $33(41.3)$ & \\
\hline \multirow{2}{*}{$\begin{array}{l}\text { Blood pressure } \\
{[\mathrm{mm} \mathrm{Hg}]}\end{array}$} & \multicolumn{2}{|c|}{ systolic } & $140.64 \pm 15.89$ & $142.42 \pm 22.36$ & 0.680 \\
\hline & \multicolumn{2}{|c|}{ diastolic } & $86.21 \pm 9.61$ & $86.97 \pm 10.75$ & 0.690 \\
\hline \multicolumn{3}{|l|}{$\begin{array}{l}\mathrm{PISA}^{\mathrm{d}}\left[\mathrm{mm}^{2}\right] \\
\text { (at baseline) }\end{array}$} & $1,772.70 \pm 580.06$ & $1,785.98 \pm 611.90$ & 0.900 \\
\hline \multicolumn{3}{|l|}{$\mathrm{BMl} \mathrm{l}^{\mathrm{a}}\left[\mathrm{kg} / \mathrm{m}^{2}\right]$} & $23.97 \pm 3.88$ & $23.23 \pm 3.83$ & 0.410 \\
\hline \multicolumn{3}{|c|}{$\begin{array}{l}\mathrm{eGFR} \text { d }\left[\mathrm{mL} / \mathrm{min} / 1.73 \mathrm{~m}^{2}\right] \\
\text { (at baseline) }\end{array}$} & $17.74 \pm 7.80$ & $19.30 \pm 9.56$ & 0.580 \\
\hline
\end{tabular}

Data presented as mean \pm standard deviation $(M \pm S D)$ or as number (percentage) ( $n(\%))$. SES - socio-economic status assessed by the Kuppuswamy scale ${ }^{10}$; CVS - cardiovascular system; CNS - central nervous system; PISA - periodontal inflamed surface area; BMI - body mass index; eGFR - estimated glomerular filtration rate; ${ }^{a} t$ test; ${ }^{b} X^{2}$ test with a continuity correction; ${ }^{c} X^{2}$ test; ${ }^{d}$ Mann-Whitney $U$ test. 
Table 2. Comparison of clinical variables within the groups across the study time points

\begin{tabular}{|c|c|c|c|c|c|c|}
\hline Group & Variables & Time point & $\min -\max$ & $M \pm S D$ & $\mathrm{Me}(I Q R)$ & $p$-valuet \\
\hline \multirow{12}{*}{ Group 1} & \multirow{3}{*}{$\begin{array}{l}\text { hs-CRP } \\
{[\mathrm{mg} / \mathrm{L}]}\end{array}$} & at baseline & $0.34-119.38$ & $6.59 \pm 17.25^{b, c}$ & $3.42(2.99)$ & \multirow{3}{*}{$<0.001^{* *}$} \\
\hline & & at 3 months & $0.42-29.30$ & $3.90 \pm 4.80^{a, c}$ & $2.80(2.09)$ & \\
\hline & & at 6 months & $0.56-15.49$ & $3.03 \pm 3.20^{a, b}$ & $2.10(1.43)$ & \\
\hline & \multirow{3}{*}{$\begin{array}{c}\text { eGFR } \\
{\left[\mathrm{mL} / \mathrm{min} / 1.73 \mathrm{~m}^{2}\right]}\end{array}$} & at baseline & $7.00-35.00$ & $17.74 \pm 7.80^{\mathrm{b}, \mathrm{c}}$ & $16.00(12.00)$ & \multirow{3}{*}{$<0.001^{* *}$} \\
\hline & & at 3 months & $8.00-39.00$ & $20.59 \pm 8.21^{a, c}$ & $20.00(12.00)$ & \\
\hline & & at 6 months & $8.00-57.00$ & $25.04 \pm 11.53^{a, b}$ & $23.00(18.00)$ & \\
\hline & \multirow{3}{*}{$\begin{array}{c}\text { UACR } \\
{[\mathrm{mg} / \mathrm{gCr}]}\end{array}$} & at baseline & $10.00-500.00$ & $184.68 \pm 209.99^{b, c}$ & $150.00(490.00)$ & \multirow{3}{*}{$<0.001^{* *}$} \\
\hline & & at 3 months & $10.00-500.00$ & $169.57 \pm 131.71^{a}$ & $75.00(140.00)$ & \\
\hline & & at 6 months & $10.00-500.00$ & $138.40 \pm 143.69^{a}$ & $75.00(125.00)$ & \\
\hline & \multirow{3}{*}{$\begin{array}{l}\text { PISA } \\
{\left[\mathrm{mm}^{2}\right]}\end{array}$} & at baseline & $1,031.12-3,506.00$ & $1,772.70 \pm 580.06^{b, c}$ & $1,679.54(617.30)$ & \multirow{3}{*}{$<0.001^{* *}$} \\
\hline & & at 3 months & $132.45-690.12$ & $279.82 \pm 119.05^{a, c}$ & 245.33 (156.19) & \\
\hline & & at 6 months & $100.21-598.65$ & $249.46 \pm 92.72^{a, b}$ & $116.43(90.27)$ & \\
\hline \multirow{12}{*}{ Group 2} & \multirow{3}{*}{$\begin{array}{l}\text { hs-CRP } \\
{[\mathrm{mg} / \mathrm{L}]}\end{array}$} & at baseline & $0.57-16.59$ & $4.80 \pm 4.22^{b, c}$ & $3.58(2.38)$ & \multirow{3}{*}{$<0.001^{* *}$} \\
\hline & & at 3 months & $0.46-20.90$ & $5.88 \pm 5.12^{a, c}$ & $3.98(4.19)$ & \\
\hline & & at 6 months & $0.44-32.23$ & $6.79 \pm 6.49^{a, b}$ & $4.32(6.43)$ & \\
\hline & \multirow{3}{*}{$\begin{array}{c}\text { eGFR } \\
{\left[\mathrm{mL} / \mathrm{min} / 1.73 \mathrm{~m}^{2}\right]}\end{array}$} & at baseline & $6.00-42.00$ & $19.30 \pm 9.56^{b, c}$ & $18.00(8.50)$ & \multirow{3}{*}{$<0.001^{* *}$} \\
\hline & & at 3 months & $6.00-53.00$ & $17.81 \pm 11.10^{\mathrm{a}, \mathrm{c}}$ & $14.00(10.00)$ & \\
\hline & & at 6 months & $4.00-45.00$ & $16.06 \pm 9.39^{a, b}$ & $15.00(9.50)$ & \\
\hline & \multirow{3}{*}{$\begin{array}{c}\text { UACR } \\
{[\mathrm{mg} / \mathrm{gCr}]}\end{array}$} & at baseline & $10.00-500.00$ & $191.52 \pm 204.86^{b, c}$ & $75.00(475.00)$ & \multirow{3}{*}{$<0.001^{* *}$} \\
\hline & & at 3 months & $10.00-500.00$ & $205.00 \pm 195.49^{a}$ & $150.00(425.00)$ & \\
\hline & & at 6 months & $10.00-500.00$ & $218.94 \pm 202.29^{a}$ & $150.00(425.00)$ & \\
\hline & \multirow{3}{*}{$\begin{array}{l}\text { PISA } \\
{\left[\mathrm{mm}^{2}\right]}\end{array}$} & at baseline & $1,062.85-3,564.15$ & $1,785.98 \pm 611.90^{b, c}$ & $1,587.84(615.08)$ & \multirow{3}{*}{$<0.001^{* *}$} \\
\hline & & at 3 months & $1,150.21-3,523.10$ & $1,819.25 \pm 599.46^{a, c}$ & 1,647.89 (662.13) & \\
\hline & & at 6 months & $1,190.22-3,594.25$ & $1,854.32 \pm 593.27^{a, b}$ & $1,696.33(658.95)$ & \\
\hline
\end{tabular}

min - minimum; max - maximum; $M$ - mean; SD - standard deviation; $M e$ - median; IQR - interquartile range; hs-CRP - high-sensitivity C-reactive protein; UACR - urine albumin-to-creatinine ratio; ${ }^{a}$ at baseline; ${ }^{b}$ at 3 months; ${ }^{c}$ at 6 months; † Friedman's test; ${ }^{*}$ highly significant $(p<0.001)$. The $95 \%$ Monte Carlo confidence interval (Cl) for group 1 was $0.00-0.06$ and for group 2 it was 0.00-0.09. Post hoc Scheffe's test applied for pairwise comparisons showed significant differences at $p<0.05$.

Table 3 and Fig. 2-5 show the intergroup comparisons of the median values of periodontal parameters, hs-CRP and renal biomarkers between the 2 groups at different time points. No statistically significant differences were observed when periodontal parameters,

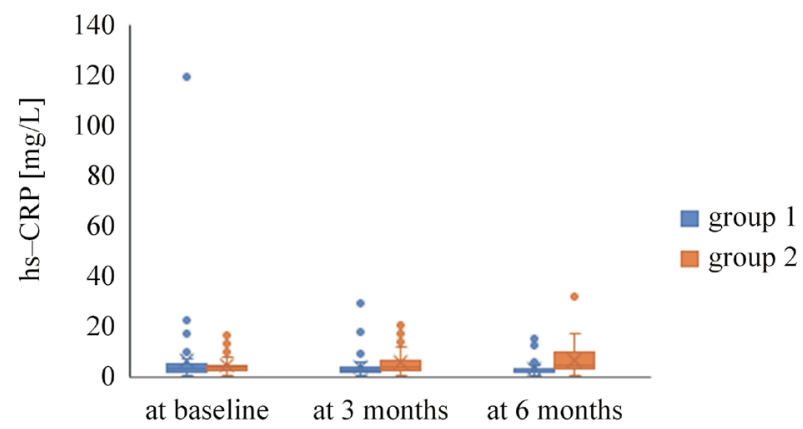

Fig. 2. Comparison of high-sensitivity C-reactive protein (hs-CRP) between the groups according to the time points

Data presented as $M \pm S D$
hs-CRP and renal biomarkers were compared between the 2 groups at baseline $(p>0.05)$. However, the abovementioned variables were statistically significantly different in the 2 groups at 3-month and 6-month followup $(p<0.05)$.

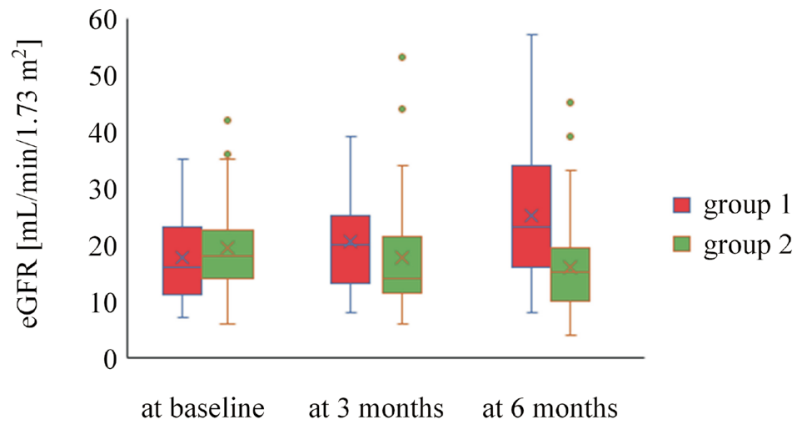

Fig. 3. Comparison of the estimated glomerular filtration rate (eGFR) between the groups according to the time points

Data presented as $M \pm S D$. 


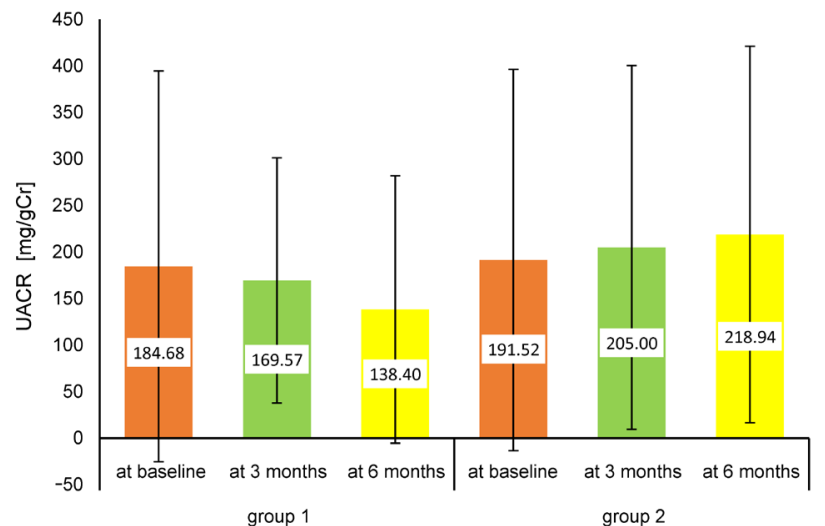

Fig. 4. Comparison of the urine albumin-to-creatinine ratio (UACR) between the groups according to the time points

Data presented as $M \pm S D$.

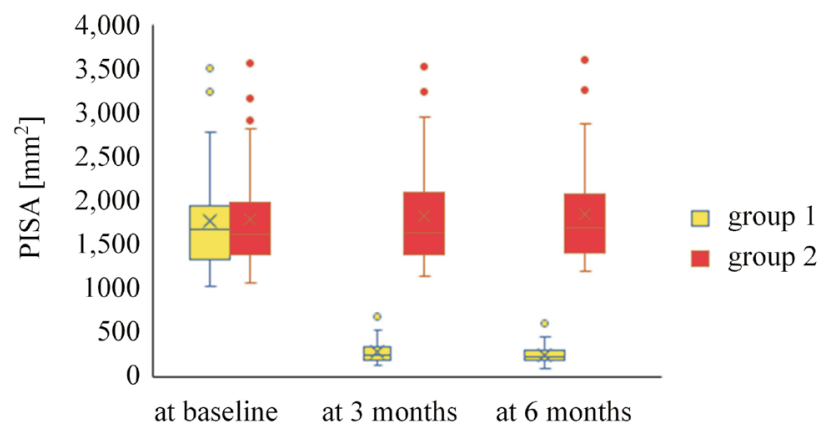

Fig. 5. Comparison of the periodontal inflamed surface area (PISA) between the groups according to the time points

Data presented as $M \pm S D$.

Table 3. Comparison of clinical variables between the groups across the study time points

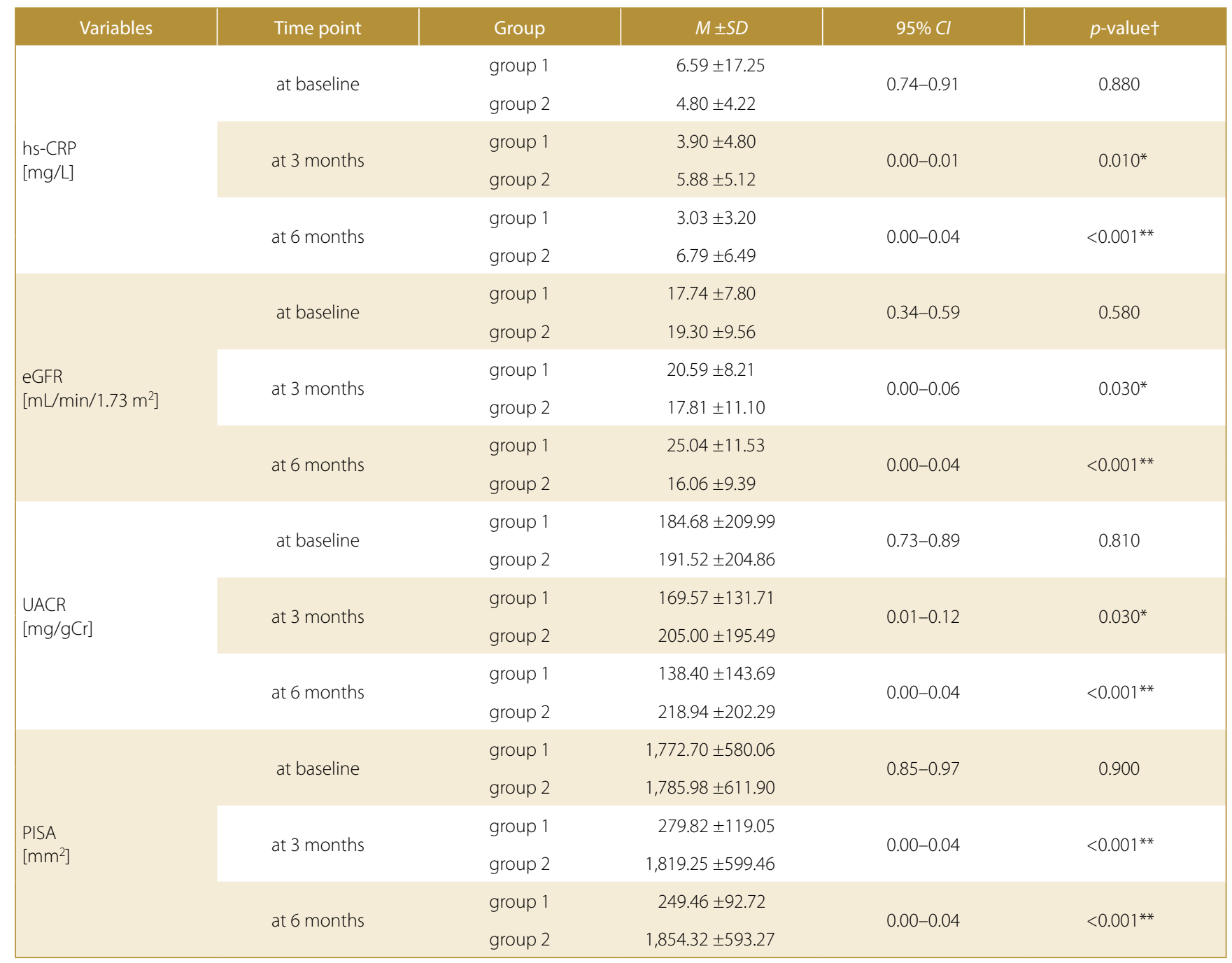

† Mann-Whitney $U$ test; ${ }^{*}$ statistically significant $(p<0.05) ;{ }^{* *}$ highly significant $(p<0.001)$.

\section{Discussion}

A recent systematic review on the association between $\mathrm{CP}$ and $\mathrm{CKD}$, and the effects of periodontal treatment concluded that there was an increased incidence of $\mathrm{CP}$ in CKD patients, with odds ratios $(O R s)$ ranging from 1.49 to $2.39 .{ }^{12}$ After multivariable adjustment, it was also found that $\mathrm{CP}$ was associated with $\mathrm{CKD}$ and that it was a modifiable 
risk factor for CKD. The authors thus emphasized the need for carefully designed interventional studies to determine whether periodontal treatment can reduce the incidence and/or severity of CKD. ${ }^{12}$

The present study was conducted to evaluate the effect of periodontal treatment on the systemic inflammatory response. The results showed a causal association between the treatment of CP and high serum levels of hs-CRP as well as high values of eGFR and UACR in CKD patients. However, there were many confounding factors, like hypertension, diabetes, smoking, and SES, leading to an increase in the systemic inflammatory burden.

In the present study, hs-CRP was used to assess the local inflammatory processes leading to the systemic inflammatory burden. It has been documented that CP induces an acute-phase inflammatory response that can be measured based on the serum level of hs-CRP, which is regarded as a key biomarker of systemic inflammation. ${ }^{13}$ High-sensitivity CRP is mainly synthesized by hepatocytes in the liver in response to inflammation and tissue damage, but can also be produced locally by arterial tissue, and is regulated by cytokines, like IL- 6 , IL- $1 \beta$ and tumor necrosis factor alpha (TNF- $\alpha){ }^{14}$

For hs-CRP, $1-3 \mathrm{mg} / \mathrm{L}$ is considered the critical range pertaining to risk prediction. Elevated levels of hs-CRP $(>2.1 \mathrm{mg} / \mathrm{L})$ are associated with a higher incidence of acute thrombotic events, including stroke and myocardial infarction. ${ }^{15}$

According to a systematic review, ${ }^{16}$ there is convincing evidence that CRP is chronically elevated in CP patients as compared to healthy controls and that it is affected by the severity of $\mathrm{CP}$, with benefits of periodontal treatment for such patients as compared to untreated ones. ${ }^{17}$

In the present study, the serum levels of hs-CRP in group 1 decreased after NSPT from $6.59 \pm 17.25 \mathrm{mg} / \mathrm{L}$ (at baseline) to $3.90 \pm 4.80 \mathrm{mg} / \mathrm{L}$ (at 3 months), and then to $3.03 \pm 3.20 \mathrm{mg} / \mathrm{L}$ (at 6 months). By contrast, in group 2, the serum levels of hs-CRP increased from $4.80 \pm 4.22 \mathrm{mg} / \mathrm{L}$ (at baseline) to $5.88 \pm 5.12 \mathrm{mg} / \mathrm{L}$ (at 3 months), and then to $6.79 \pm 6.49 \mathrm{mg} / \mathrm{L}$ (at 6 months). D'Aiuto et al. reported that the serum levels of CRP were reduced in 65 patients with severe $\mathrm{CP}$, otherwise systemically healthy, who were subjected to intensive NSPT for 2 months. ${ }^{18}$ Vilela et al. reported a reduction in the levels of prohepcidin, IL-6 and hs-CRP in both CKD and control patients following periodontal treatment. ${ }^{19}$ Their findings suggest that CP induces the systemic inflammatory response and is more severe in CKD patients; however, successful periodontal treatment reduces the inflammatory burden in such patients, and thus may be a beneficial intervention in the therapy of CKD. ${ }^{19}$ In a study by Guo and Lin, 53 patients were divided into 3 groups: group A received treatment for both CKD and CP; group B received treatment for CKD only; and group $\mathrm{C}$ received treatment for $\mathrm{CP}$ only..$^{20}$ The authors concluded that in patients with CKD and CP, NSPT decreased both the clinical parameters of $\mathrm{CP}$ and the serum markers of systemic inflammation. The levels of hs-CRP were reduced in all groups, especially in group A, with a decrease from $4.71 \pm 1.55 \mathrm{mg} / \mathrm{L}$ pre-operatively to $3.70 \pm 1.28 \mathrm{mg} / \mathrm{L}$ post-operatively. ${ }^{20}$ Elevated levels of CRP are also related to several other CKD risk factors, such as SES, old age, prolonged diabetes, CVD, and albuminuria; however, periodontitis is an independent predictor of increased hs-CRP in CKD patients. ${ }^{21}$ Considering the presence of similar confounding factors in both groups in the present study, a decrease in hs-CRP in group 1 can be attributed to NSPT only, wherein patient motivation and counseling by the periodontist during treatment led to psychological benefits, an increased patient understanding of the need to maintain good oral hygiene, and increased treatment compliance with regard to both CKD and CP.

In this study, eGFR and UACR were used as renal biomarkers to evaluate the progression or regression of CKD. There are many methods to diagnose and stage CKD. The estimated GFR is used for diagnosis, staging and prognosis. It also plays an important role in drug dosing and risk stratification for clinical procedures and future outcomes. The estimated GFR is usually calculated based on the plasma creatinine values, using the Modification of Diet in Renal Disease (MDRD) Study equation or the Chronic Kidney Disease Epidemiology Collaboration (CKD-EPI) equation. ${ }^{22}$ In the present study, the MDRD formula was used to calculate eGFR. This formula has been extensively validated in multiple samples with and without CKD. Many of the studies have shown a good performance of the method in CKD patients, including those with diabetes or kidney transplants, and the elderly. ${ }^{23}$ Albuminuria is the most frequently used marker of kidney damage in clinical practice and research. Over $50 \%$ of CKD cases may be missed if UACR is ignored. The estimation of UACR has advantages over other tests in terms of sensitivity and the level quantification. Moreover, the assessment of UACR in CKD screening appears to enable a more accurate prediction of poor quality of life. ${ }^{24}$

The results of the present study are similar to those obtained by Almeida et al. ${ }^{25}$ The authors reported significant improvement after an intervention in the median (25\%, 75\% percentiles) eGFR values from 34.6 $(27.0,44.7) \mathrm{mL} / \mathrm{min} / 1.73 \mathrm{~m}^{2}$ at baseline to $37.6(29.7$, $57.0) \mathrm{mL} / \mathrm{min} / 1.73 \mathrm{~m}^{2}$ on day 90 and $37.6(28.6,56.0)$ $\mathrm{mL} / \mathrm{min} / 1.73 \mathrm{~m}^{2}$ on day $180 .^{25}$

Carillo Artese et al. evaluated the effect of NSPT with no antibiotics on the levels of creatinine and eGFR at baseline and 3 months after treatment in 21 pre-dialysis CKD patients and 19 individuals without CKD. ${ }^{26}$ Both groups showed significant and similar post-treatment improvement in all parameters examined. ${ }^{26}$ Periodontal treatment may thus play an important role in reducing inflammatory mediators. Several studies have indicated that the kidneys are important for the clearance of cytokines. ${ }^{27}$ High levels of inflammatory markers may have detrimental effects on the renal system, because an increased nephron filtration 
of plasma proteins results in further release of cytokines in the renal interstitium, with subsequent fibroblast proliferation, fibrogenesis, and ultimately renal scarring, contributing to progressive deterioration in function. Hence, periodontal treatment could have positive effects on both systemic inflammatory markers and renal endothelial function via more effective filtration. ${ }^{28}$

The present study used PISA as the main periodontal parameter. Periodontal inflammation, which occurs locally, but can disseminate systemically, is the biological basis for the plausibility of any potential association between $\mathrm{CP}$ and other diseases. In this sense, any classification of $\mathrm{CP}$ as a potential risk factor for any systemic condition should quantify the inflammatory burden posed by periodontitis. ${ }^{29}$ For this reason, the PISA classification, which was developed in 2008, is based not only on linear measurements, such as CAL and gingival recession (GR), but also on BoP. Thus, PISA reflects the surface area of bleeding pocket epithelium in square millimeters. ${ }^{4}$ Notably, PISA is considered by medical professionals other than dentists as a useful index for understanding the degree of inflammation induced by PD. As stated by Leira et al., a PISA value $\geq 130.33 \mathrm{~mm}^{2}$ indicates CP while values ranging from $934.71 \mathrm{~mm}^{2}$ to $3,274.96 \mathrm{~mm}^{2}$ indicate severe periodontitis cases according to the Centers for Disease Control and Prevention and American Academy of Periodontology (CDC-AAP) classification. ${ }^{29}$ A study evaluating the dose-response relationship between PISA and the level of $\mathrm{HbA} 1 \mathrm{c}$ revealed that PISA indeed appeared to be a valuable tool for assessing the relationships between the amount of inflamed periodontal tissue and well-defined disease activity parameters, such as HbA1c. ${ }^{30}$ To date, one Japanese study has used this index in patients with CKD and CP. ${ }^{31}$ The study investigated the effect of PD on kidney function in community-dwelling elderly patients. Participants were classified into quartile groups according to their PISA score, and then divided into 2 groups: the highest quartile vs. the other 3 groups combined. The results showed that patients in the highest PISA quartile had a 2.6-fold greater risk of a decreased kidney function after 2 years of follow-up (OR: 2.58; 95\% confidence inter$\operatorname{val}(C I): 1.34-4.98) .{ }^{31}$

The results of the present study differ from those of the Kidney and Periodontal Disease (KAPD) study. ${ }^{32}$ In the KAPD study, there was no change in the magnitude of the biomarkers measured in response to intensive periodontal treatment. There are multifactorial potential reasons for this difference, including the presence of more severe PD in the immediate treatment group, the lack of a true control group, and the fact that the rescue group in the KAPD study received oral hygiene instructions, cleaning above the gum line, and had hopeless teeth extracted, all of which could have reduced the burden of PD, thus reducing the ability to achieve a clear separation in terms of periodontal status between groups and to detect differences in biomarkers. ${ }^{32}$
Persistent low-grade inflammation has been recognized as an essential component of CKD, and so CP may contribute to this inflammation. The pathogenesis of CP involves polymicrobial synergy and dysbiosis (the PSD model), leading to a greater relative abundance of individual components of the bacterial community as compared to their abundance in health, with alterations in the host-microbe crosstalk sufficient to mediate destructive inflammation and bone loss. ${ }^{33}$ Among all periodontal pathogens, the role of Porphyromonas gingivalis ( $P$. gingivalis) is the most powerful in this respect. Hence, in PD, a larger area of the inflamed periodontal surface may allow this keystone periodontal pathogen and its byproducts, like gingipains and fimbriae, to enter circulation through an ulcerated periodontal pocket, inducing bacteremia. ${ }^{34}$ In turn, this appears to activate cells, series of receptors and signaling pathways, e.g., dendritic cells, which are the critical cells of the immune response and the 'presenters' of antigens that can also serve as the 'transporters' of bacteria and their virulence factors. ${ }^{34}$ Moreover, gingipains from $P$. gingivalis have been shown to activate protease-activated receptors (PARs) and toll-like receptors (TLRs), leading to an increased inflammatory response with the release of chemokines $^{35}$ and pro-inflammatory cytokines, like IL-6, prostaglandin E2 (PGE2) and thromboxane B2 (TXB2), in the kidneys, generating an inflammatory cascade that can lead to kidney dysfunction. These factors may accelerate angiogenesis, thrombus formation and platelet aggregation via several mechanisms causing vasoconstriction and a chronic reduction in renal blood flow. The atherogenesis of the renal artery and arterioles can also cause ischemia, glomerular sclerosis and severe renal insufficiency. ${ }^{36}$

Alternatively, the effect of periodontitis on renal tissue can be explained by the concept of oral microbial pathogens affecting distant organs directly through bloodstream dissemination, a 'mobile oral microbiome', involving the direct migration of resident oral microbial species and their colonization in distant organs. ${ }^{37}$ In some case studies, ${ }^{38}$ these oral pathogens have been directly indicated as a cause of bacterial endocarditis, acute post-streptococcal glomerulonephritis and infective endocarditisassociated glomerulonephritis. The $P$. gingivalis fimbriae bind to cluster of differentiation 14 (CD14) and activate TLR2-phosphoinositide 3-kinase (PI3K) signaling. The activated TLR2 pro-adhesive signaling pathway enhances leukocyte-endothelial cell interactions, transendothelial migration and the interaction of its cell surface fimbriae with complement receptor 3 (CR3). The activated CR3 then downregulates IL-12p70, a key cytokine involved in macrophage phagocytosis. This CR3-associated reduction in IL-12p70 impairs the clearance of $P$. gingivalis and promotes its survival in renal tissue. ${ }^{39}$

At the molecular level, hsa-miR-146a, hsa-miR-146b, hsa-miR-155, and hsa-miR200 could be considered as potential biomarkers of the progression of PD. ${ }^{40}$ To date, only an animal study with transcription factor 
signal transducer and activator of transcription (STAT) has been carried out considering both PD and CKD. ${ }^{41}$ The study demonstrated that STAT1 expression was augmented by periodontitis, and resulted in the upregulation of inflammatory and fibrosis genes, which aggravated hypertensive renal injuries in the mouse model, thus suggesting a new target for periodontal treatment in CKD patients. ${ }^{41}$

Notably, $30-50 \%$ of both ESRD patients on RRT and non-dialyzed CKD patients show evidence of an active systemic inflammatory response in the form of elevated serum levels of CRP and other pro-inflammatory cytokines, like IL-1 and IL-6. ${ }^{42}$ Therefore, there are 2 potential mechanisms through which the immune system and the inflammatory process may affect distant organs, including the kidneys. However, the mechanisms through which CP influences CKD are not well understood. This process is not likely to involve a single causal mechanism, but rather a combination of mechanisms.

The present study is a novel effort to evaluate the effect of NSPT on the PISA scores in patients with CKD and CP. An increase in the PISA scores, as observed in group 2 in our study, can be explained by the high prevalence and severity of periodontitis in CKD patients. An in-depth review by Akar et al. explored the etiological factors and systemic consequences of poor oral health in CKD patients, and observed that oral hygiene was generally poor in such patients, which might cause inflammation, infection and atherosclerosis, and could worsen their condition. ${ }^{43}$ Hence, maintaining good oral health is essential for such patients, and should be monitored both by nephrologists and periodontists.

Apart from NSPT, periodontal therapy can include laser therapy (LT) and photodynamic therapy (PDT) as methods of antimicrobial treatment to kill periodontal bacteria with few adverse effects. The abovementioned alternatives can be used as independent treatment or as an adjunct to SRP. A randomized clinical trial evaluating all 3 treatment modalities reported changes in the expression of 13 different cytokines, i.a., IL-1 and IL-6, and 9 acute-phase proteins, i.a., CRP, in the gingival crevicular fluid (GCF) of CP patients. ${ }^{44}$

The present study is the pilot study of an ongoing longitudinal prospective trial, wherein the correlation between CKD and the treatment of CP will be evaluated through the multivariate logistic regression analysis. Some limitations of the present study are as follows: the patient population was taken from a single center; some patients dropped out; and the frequency of use of antihypertensive drugs and their effects on renal biomarkers were not examined. These limitations should be addressed in future studies.

\section{Conclusions}

Chronic periodontitis is a non-traditional risk factor for CKD that can be treated in a dental office by means of NSPT and proper maintenance visits, which are expected to reduce the inflammatory burden, and thus limit the effect of contributing factors on further progression of CKD. Therefore, methods of early CP detection, risk factors for kidney disease and specifically dental interventions warrant attention from global public health organizations. In the future, larger studies using different biomarkers and long-term follow-up are needed to evaluate the contribution of periodontal treatment in reducing the risk of $\mathrm{CKD}$ or its progression.

\section{ORCID iDs}

Kirti Suresh Vachhani (1) https://orcid.org/0000-0002-0358-0712 Neeta Vijay Bhavsar (10 https://orcid.org/0000-0002-4379-3376

\section{References}

1. Kane SF. The effects of oral health on systemic health. Gen Dent. 2017;65(6):30-34. PMID:29099363.

2. Frencken JE, Sharma P, Stenhouse L, Green D, Laverty D, Dietrich T. Global epidemiology of dental caries and severe periodontitis - a comprehensive review. J Clin Periodontol. 2017;44(Suppl 18):S94-S105. doi:10.1111/jcpe.12677

3. Sharma P, Cockwell P, Dietrich T, Ferro C, Ives N, Chapple ILC. INfluence of Successful Periodontal Intervention in REnal Disease (INSPIRED): Study protocol for a randomised controlled pilot clinical trial. Trials. 2017;18:535. doi:10.1186/s13063-017-2236-5

4. Nesse W, Abbas F, van Der Ploeg I, Spijkervet FKL, Dijkstra PU, Vissink A. Periodontal inflamed surface area: Quantifying inflammatory burden. J Clin Periodontol. 2008;35(8):668-673. doi:10.1111/j.1600-051X.2008.01249.x

5. Sabharwal A, Gomes-Filho IS, Stellrecht E, Scannapieco FA. Role of periodontal therapy in management of common complex systemic diseases and conditions: An update. Periodontol 2000. 2018;78(1):212-226. doi:10.1111/prd.12226

6. Bikbov B, Purcell CA, Levey AS, et al.; GBD Chronic Kidney Disease Collaboration. Global, regional, and national burden of chronic kidney disease, 1990-2017: A systematic analysis for the Global Burden of Disease Study 2017. Lancet. 2020;395(10225):709-733. doi:10.1016/S0140-6736(20)30045-3

7. Kasiske $\mathrm{BL}$, Wheeler $\mathrm{DC}$. Kidney disease: Improving global outcomes - an update. Nephrol Dial Transplant. 2014;29(4):763-769. doi:10.1093/ndt/gft441

8. Chambrone L, Foz AM, Guglielmetti MR, et al. Periodontitis and chronic kidney disease: A systematic review of the association of diseases and the effect of periodontal treatment on estimated glomerular filtration rate. J Clin Periodontol. 2013;40(5):443-456. doi:10.1111/jcpe.12067

9. Zhao D, Khawaja AT, Jin L, Li KY, Tonetti M, Pelekos G. The directional and non-directional associations of periodontitis with chronic kidney disease: A systematic review and meta-analysis of observational studies. J Periodontal Res. 2018;53(5):682-704. doi:10.1111/jre.12565

10. Shaikh Z, Pathak R. Revised Kuppuswamy and B G Prasad socioeconomic scales for 2016. Int J Community Med Public Healh. 2017;4(4):997-999. doi:10.18203/2394-6040.ijcmph20171313

11. Greene JC, Vermillon JR. The Simplified Oral Hygiene Index. J Am Dent Assoc. 1964;68:7-13. doi:10.14219/jada.archive.1964.0034

12. Deschamps-Lenhardt S, Martin-Cabezas R, Hannedouche T, Huck O. Association between periodontitis and chronic kidney disease: Systematic review and meta-analysis. Oral Dis. 2019;25(2):385-402. doi:10.1111/odi.12834

13. Panichi V, Migliori M, De Pietro S, et al. The link of biocompatibility to cytokine production. Kidney Int. 2000;58(Suppl 76):S96-S103. doi:10.1046/j.1523-1755.2000.07612.x

14. Bansal T, Pandey A, Deepa D, Asthana AK. C-reactive protein (CRP) and its association with periodontal disease: A brief review. J Clin Diagn Res. 2014;8(7):ZE21-ZE24. doi:10.7860/JCDR/2014/8355.4646

15. Ridker PM, Rifai N, Rose L, Buring JE, Cook NR. Comparison of C-reactive protein and low-density lipoprotein cholesterol levels in the prediction of first cardiovascular events. $N$ Engl J Med. 2002;347(20):1557-1565. doi:10.1056/NEJMoa021993 
16. Paraskevas $S$, Huizinga JD, Loos BG. A systematic review and metaanalyses on C-reactive protein in relation to periodontitis. $J$ Clin Periodontol.2008;35(4):277-290. doi:10.1111/j.1600-051X.2007.01173.x

17. Alade GO, Ayanbadejo PO, Umeizudike KA, Ajuluchukwu JN. Association of elevated C-reactive protein with severe periodontitis in hypertensive patients in Lagos, Nigeria: A pilot study. Contemp Clin Dent. 2018;9(Suppl 1):S95-S99. doi:10.4103/ccd.ccd_104_18

18. D'Aiuto F, Nibali L, Parkar M, Suvan J, Tonetti MS. Short-term effects of intensive periodontal therapy on serum inflammatory markers and cholesterol. J Dent Res. 2005;84(3):269-273. doi:10.1177/154405910508400312

19. Vilela EM, Bastos JA, Fernandes N, Ferreira AP, Chaoubah A, Bastos MG. Treatment of chronic periodontitis decreases serum prohepcidin levels in patients with chronic kidney disease. Clinics (Sao Paulo). 2011;66(4):657-662. doi:10.1590/S1807-59322011000400022

20. Guo N, Lin G. Effects of nonsurgical periodontal therapy on serum inflammatory factor levels in patients with chronic kidney disease and periodontitis. Biomed Res. 2017;28(9):3899-3902. https://www.biomedres.info/biomedical-research/effects-ofnonsurgical-periodontal-therapy-on-serum-inflammatory-factorlevels-in-patients-with-chronic-kidney-disease-and-periodo.pdf. Accessed October 22, 2019.

21. loannidou $E$, Swede $H$, Dongari-Bagtzoglou A. Periodontitis predicts elevated C-reactive protein levels in chronic kidney disease. J Dent Res. 2011;90(12):1411-1415. doi:10.1177/0022034511423394

22. Matsushita K, Mahmoodi BK, Woodward M, et al. Comparison of risk prediction using the CKD-EPI equation and the MDRD study equation for estimated glomerular filtration rate. JAMA. 2012;307(18):1941-1951. doi:10.1001/jama.2012.3954

23. Levey AS, Coresh J, Greene T, et al. Using standardized serum creatinine values in the modification of diet in renal disease study equation for estimating glomerular filtration rate. Ann Intern Med. 2006;145(4):247-254. doi:10.7326/0003-4819-145-4-200608150-00004

24. Park Jl, Baek H, Kim BR, Jung HH. Comparison of urine dipstick and albumin: Creatinine ratio for chronic kidney disease screening: A population-based study. PLoS One. 2017;12(2):e0171106. doi:10.1371/journal.pone.0171106

25. Almeida S, Figueredo CM, Lemos C, Bregman R, Fischer RG. Periodontal treatment in patients with chronic kidney disease: A pilot study. J Periodontal Res. 2017;52(2):262-267. doi:10.1111/jre.12390

26. Carillo Artese HP, Oliveira de Sousa C, Luiz RR, Sansone C, Medeiros de Barros Torres MC. Effect of non-surgical periodontal treatment on chronic kidney disease patients. Braz Oral Res. 2010;24(4):449-454. doi:10.1590/S1806-83242010000400013

27. Craig RG. Interactions between chronic renal disease and periodontal disease. Oral Dis. 2008;14(1):1-7. doi:10.1111/j.1601-0825.2007.01430.x

28. Graziani F, Cei S, La Ferla F, Vano M, Gabriele M, Tonetti M. Effects of non-surgical periodontal therapy on the glomerular filtration rate of the kidney: An exploratory trial. J Clin Periodontol. 2010;37(7):638-643. doi:10.1111/j.1600-051X.2010.01578.x

29. Leira Y, Martín-Lancharro P, Blanco J. Periodontal inflamed surface area and periodontal case definition classification. Acta Odontol Scand. 2018;76(3):195-198. doi:10.1080/00016357.2017.1401659

30. Nesse W, Linde A, Abbas F, et al. Dose-response relationship between periodontal inflamed surface area and $\mathrm{HbA1c}$ in type 2 diabetics. J Clin Periodontol. 2009;36(4):295-300. doi:10.1111/j.1600-051X.2009.01377.x

31. Iwasaki M, Taylor GW, Nesse W, Vissink A, Yoshihara A, Miyazaki H. Periodontal disease and decreased kidney function in Japanese elderly. Am J Kidney Dis. 2012;59(2):202-209. doi:10.1053/j.ajkd.2011.08.027

32. Grubbs V, Garcia F, Vittinghoff E, et al. Nonsurgical periodontal therapy in CKD: Findings of the Kidney and Periodontal Disease (KAPD) pilot randomized controlled trial. Kidney Med. 2020;2(1):49-58. doi:10.1016/j.xkme.2019.09.005

33. Hajishengallis G. Immunomicrobial pathogenesis of periodontitis: Keystones, pathobionts, and host response. Trends Immunol. 2014;35(1):3-11. doi:10.1016/j.it.2013.09.001

34. Hasturk $H$, Kantarci A. Activation and resolution of periodontal inflammation and its systemic impact. Periodontol 2000. 2015;69(1):255-273. doi:10.1111/prd.12105

35. Palm E, Demirel I, Bengtsson T, Khalaf $H$. The role of toll-like and protease-activated receptors in the expression of cytokines by gingival fibroblasts stimulated with the periodontal pathogen Porphyromonas gingivalis. Cytokine. 2015;76(2):424-432. doi:10.1016/j.cyto.2015.08.263
36. Kshirsagar AV, Moss KL, Elter JR, Beck JD, Offenbacher S, Falk RJ. Periodontal disease is associated with renal insufficiency in the Atherosclerosis Risk In Communities (ARIC) study. Am J Kidney Dis. 2005;45(4):650-657. doi:10.1053/j.ajkd.2004.12.009

37. Naghsh N, Sabet NK, Vahidi F, Mogharehabed A, Yaghini J. Relationship between periodontal disease and serum factors in patients undergoing hemodialysis. Open Dent J. 2018;11:701-709. doi:10.2174/1874210601711010701

38. Hickey NA, Shalamanova L, Whitehead KA, Dempsey-Hibbert N, van der Gast C, Taylor RL. Exploring the putative interactions between chronic kidney disease and chronic periodontitis. Crit Rev Microbiol. 2020;46(1):61-77. doi:10.1080/1040841X.2020.1724872

39. Chopra A, Sivaraman K. An update on possible pathogenic mechanisms of periodontal pathogens on renal dysfunction. Crit Rev Microbiol. 2019;45(5-6):514-538. doi:10.1080/1040841X.2018.1553847

40. Cuevas-González MV, Suaste-Olmos F, García-Calderón AG, et al. Expression of microRNAs in periodontal disease: A systematic review. Biomed Res Int. 2021;2021:2069410. doi:10.1155/2021/2069410

41. Yang $\mathrm{Q}$, Ding $\mathrm{H}$, Wei $W$, et al. Periodontitis aggravates kidney injury by upregulating STAT1 expression in a mouse model of hypertension. FEBS Open Bio. 2021;11(3):880-889. doi:10.1002/2211-5463.13081

42. Ismail G, Dumitriu HT, Dumitriu AS, Ismail FB. Periodontal disease: A covert source of inflammation in chronic kidney disease patients. Int J Nephrol. 2013;2013:515796. doi:10.1155/2013/515796

43. Akar H, Akar GC, Carrero JJ, Stenvinkel P, Lindholm B. Systemic consequences of poor oral health in chronic kidney disease patients. Clin J Am Soc Nephrol. 2011;6(1):218-226. doi:10.2215/CJN.05470610

44. Giannopoulou C, Cappuyns I, Cancela J, Cionca N, Mombelli A. Effect of photodynamic therapy, diode laser, and deep scaling on cytokine and acute-phase protein levels in gingival crevicular fluid of residual periodontal pockets. J Periodontol. 2012;83(8):1018-1027. doi:10.1902/jop.2011.110281 